\title{
$\beta 1$-adrenoceptor Arg389Gly polymorphism and heart disease: marching toward clinical practice integration
}

\author{
"...it is quite plausible that, soon, $\beta_{1} A R$ genotyping of the \\ cardiovascular patient for the Arg389Gly variation will allow for \\ personalization of his/her drug therapy."
}

Keywords: $\beta_{1}$-adrenergic receptor $\bullet \beta$-blocker $\bullet$ cardiovascular disease $\bullet$ dobutamine $\bullet$ drug response $\bullet$ GWAS $\bullet$ heart failure $\bullet$ hypertension $\bullet$ polymorphism

The $\beta_{1}$-adrenergic receptor (AR) is a G protein-coupled receptor (GPCR) abundantly expressed in the plasma membranes of human cardiac myocytes. It is the primarily responsible AR for mediating the catecholamineinduced positive inotropy and chronotropy in the heart [1]. The human $\beta_{1} \mathrm{AR}$ gene ( $A d r b 1)$ encodes a 477-amino acid functional protein through a nucleotide sequence that has at least nine nonsynonymous variations reported to date. Out of these, the Gly/Arg polymoprhism at amino acid position 389 (cytosine or guanine at nucleotide 1165 of its coding region) is by far the best-characterized variation to date affecting function and signaling of the encoded receptor $[2,3]$. This amino acid position is located in the intracellular C-terminus of the $\beta_{1} \mathrm{AR}$, a region that purportedly participates in the receptor's interactions with the $G_{s}$ protein, and also gets phosphorylated by GPCR-kinases (GRKs), which induces the subsequent binding of $\beta$-arrestins that terminates G-protein signaling (receptor desensitization) [1]. Consequently, this $\mathrm{C}$-terminal region is crucial for both activation and termination of the $\beta_{1}$ AR-dependent pro-contractile signaling in the heart [1]. Indeed, the Arg variant, substantially less prevalent in African Americans than in Caucasians ( 58 vs $73 \%$, respectively), is presumed to favor/stabilize the active state of the human $\beta_{1} A R$ [2], rendering the $\operatorname{Arg} 389$ $\beta_{1} \mathrm{AR}$ hyperfunctional compared with the Gly389 receptor. In vitro studies in heterologous cell systems (cell lines with the two variants exogenously overexpressed) have confirmed the higher activity of the Arg389 receptor in terms of second messenger cAMP (cyclic adenosine monophosphate) accumulation, Gs protein coupling efficiency and high affinity ligand binding in the absence of guanine triphosphate (GTP) $[2,3]$. Accordingly, it has also been found to display enhanced homologous (agonist-induced) desensitization versus the Gly variant [4]. In physiologically relevant cell systems, in other words, murine cardiomyocytes isolated from cardiac-specific overexpressors of the two variants, increased contractility and receptor desensitization (in old age) were displayed by the Arg variant-overexpressing mice [5], and the beating frequency of cardiomyocytes isolated from these mice was also higher than that of Gly $\beta_{1} \mathrm{AR}$-overexpressing myocytes [6]. However, studies on the native variant receptors in explanted human heart tissue failed to confirm differences in activity/signaling between the two variants, possibly because the human heart tissue studied was not normal (explanted from heart failure patients) [4]. Thus, the overall cardiac status may very well affect or mask the functional status of the polymorphic $\beta_{1} \mathrm{AR}$ [7]. Of course, inter-species differences (murine vs human) in cardiomyocyte physiology and cardiac $\beta_{1}$ AR biochemistry might be another reason for these discrepancies. Finally, the agonist-activated $\operatorname{Arg} 389$ variant was recently shown to undergo GRK-dependent hyperphosphorylation leading to enhanced $\beta$-arrestin2 binding (relative to the Gly $\beta_{1} \mathrm{AR}$ ) in heterologous HEK293 cells in vitro,

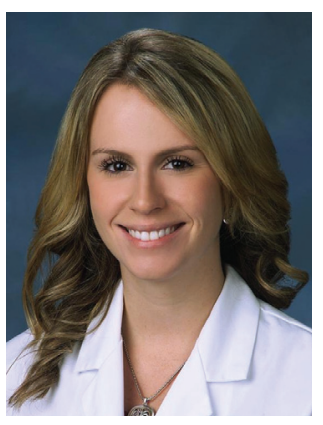

Katie A McCrink Laboratory for the Study of Neurohormonal Control of the Circulation, Department of Pharmaceutical Sciences, Nova Southeastern University College of Pharmacy, Ft Lauderdale, FL, USA

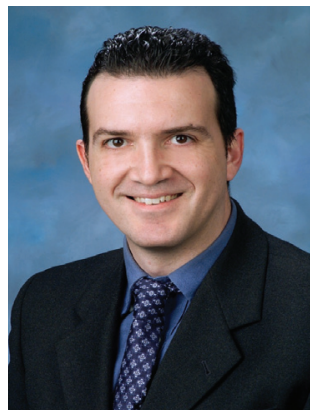

Anastasios Lymperopoulos Author for correspondence: Laboratory for the Study of Neurohormonal Control of the Circulation, Department of Pharmaceutical Sciences, Nova Southeastern University College of Pharmacy, 3200 S. University Dr., HPD (Terry) Bldg/Room 1338, Ft Lauderdale, FL 33328-2018, USA Tel.: +1 9542621338 Fax: +1 9542622278 al806@nova.edu




consistent with the elevated homologous desensitization of the $\operatorname{Arg} 389$ variant [8]. On the other hand, the agonist-activated $\operatorname{Arg} 389 \beta_{1}$ AR seems to preferentially bind $\beta$-arrestin 2 over $\beta$-arrestin1 when overexpressed in neonatal rat ventricular myocytes (NRVMs), while the Gly389 $\beta_{1}$ AR shows no preference for either of the two $\beta$-arrestins (Lymperopoulos A, Unpublished DAтA). Given that $\beta$-arrestin2-dependent signaling in the heart may very well be beneficial for cardiac function, contrary to the cardiotoxic and negative for contractility $\beta$-arrestin1-dependent signaling [9], this preference of the Arg variant for $\beta$-arrestin2 interaction in NRVMs may underlie the hyperactivity of the Arg389 $\beta_{1} \mathrm{AR}$ toward elevated contractility/cardiac function. This finding also suggests that the $\beta$-arrestin $1 / 2$ (and GRK) complements of the human cardiomyocyte, available for interaction with the $\beta_{1} \mathrm{AR}$, must be taken into account when studying the cardiac effects of the Arg389Gly $\beta_{1}$ AR polymorphism.

\section{"Quite frequently in pharmacogenomics, small candidate gene association studies report exciting and promising results, which soon turn out to be biased, false positive and irreproducible in larger GWAS's."}

Given that the $\beta_{1} A R$ is mainly expressed in the heart, where it stimulates cardiac output, and in the juxtaglomerular apparatus of the kidneys, where it induces renin release activating the renin-angiotensin-aldosterone system (RAAS) [2], it comes as no surprise that most studies have focused on associations of the Arg389Gly polymorphism with heart failure and hypertension. Indeed, large cohort studies have indicated that the Arg allele is associated with hypertensive risk, while the Gly allele confers lower risk for (essential) hypertension [10]. However, larger (in $>1000$ subjects) genome-wide association studies (GWAS) have failed to confirm this association [2]. Likewise, studies exploring heart failure association, albeit smaller than those on hypertension risk, have also failed to consistently link the Arg389Gly variation (or any other $A d r b 1$ locus variation) with risk for heart failure [2]. Nonetheless, this polymorphism has been associated with birth weight in Europeans, which may play a role in hypertension development later in adulthood [11]. Finally, other cardiovascular conditions potentially associated with the Arg389Gly polymorphism include acute STsegment elevation myocardial infarction (STEMI) [12], idiopathic dilated cardiomyopathy (DCM) in a small Mexican population [13], arrhythmogenesis in Japanese DCM patients [14] and small vessel disease underlying ischemic stroke in a North Indian population [15]. Of course, these associations await confirmation in large GWAS's as well as mechanistic delineation in functional in vitro studies.

With regards to drug response, the Arg389 homozygotes exhibit larger responses to the $\beta_{1}$ AR-agonist and positive inotrope drug dobutamine than Gly carriers, in terms of both cardiac function and plasma renin activity [16-18]. Similar observations have been made for several $\beta$-blockers ( $\beta_{1}$ AR-antagonists): Arg389 carriers show greater reductions in cardiac hemodynamic parameters, heart rate, blood pressure and plasma renin activity, in response to various $\beta$-blockers (metoprolol, bisoprolol, esmolol, atenolol) than Gly carriers [2]. This is consistent with the $\operatorname{Arg} 389 \beta_{1} \mathrm{AR}$ being more responsive to another $\beta$-blocker, carvedilol, in vitro [6], and suggests that the Arg allele normally, in other words, in healthy carriers, confers more robust responses to $\beta$-blocker drugs. Nevertheless, the majority of studies on the effects of this polymorphism on $\beta$-blocker responses are in heart failure patients, as expected, since $\beta$-blockers are nowadays part of the mainstay chronic heart failure drug regimen [9]. Consistent with reports from normal individuals, $\beta$-blocker-treated homozygous Arg389 patients show substantially larger improvements in their left ventricular indices than Gly389carrying patients treated with the same drug [19]. In addition, important genetic subgroup studies of the BEST ( $\beta$-blocker Evaluation of Survival) clinical trial have provided convincing evidence for better efficacy of the $\beta$-blocker bucindolol (pharmacologically very similar to carvedilol) in Arg389 homozygotes, especially if the Ins322-325Del Adra2c gene (the gene for the $\alpha_{2 \mathrm{C}} \mathrm{AR}$ ) variant co-exists [20].

In summary, the more common (thereby considered (wild type') Arg389 allele appears to encode a $\beta_{1} A R$ that, compared with its Gly389 counterpart, is hyperfunctional at the molecular and signaling levels, and quite likely at the physiological level (i.e., in the cardiovascular system), as well. This seems to translate into better responses of the Arg allele carriers (both healthy individuals and patients) to $\beta_{1} \mathrm{AR}$ ligand drugs, either agonists (dobutamine) or antagonists ( $\beta$-blockers). In contrast, whether the Arg389Gly variation influences cardiovascular disease risk (mainly, heart failure or hypertension) remains an open question. Several obstacles need to be overcome by future studies in order to reach some consensus on addressing this question. First and foremost, the sample size of the association studies needs to be as large as possible. Quite frequently in pharmacogenomics, small candidate gene association studies report exciting and promising results, which soon turn out to be biased, false positive and irreproducible in larger GWAS's. In fact, the rule of thumb appears to be that the sample size 
of a study and its likelihood of confirming an association, are inversely proportional. The recent incorporation of very large consortia, in the order of several thousands of individuals, in the design of GWAS's will undoubtedly increase both the sensitivity and specificity of the reported associations in the future. Apart from sample size considerations and from taking the much more scientifically rigorous GWAS, instead of the candidate gene study, approach, another big roadblock is the multitrait status/complexity of most diseases (especially heart diseases). Heart failure and hypertension are extremely multifaceted disease entities, develop over long periods of time and their pathophysiologies involve hundreds (maybe even thousands) of genes. Moreover, the disease state itself may reciprocally modify the pathophysiological effects of the polymorphism (e.g., different effects of the Arg389Gly $\beta_{1}$ AR polymorphism in healthy vs diseased myocardium, see above). Additional complicating factors are the potential for linkage disequilibrium (i.e., involvement in a haplotype) of the studied polymorphism (the Arg389Gly variation is in linkage disequilibrium with the Ser49Gly variation, also within the Adrb1 coding region) and the 'dosage' effect of the polymorphism, in other words, whether the phenotypic effects of the two variants are equalized by each other and the polymorphism requires both alleles to manifest itself. This may very well be the case also with the $\operatorname{Arg} 389 \beta_{1}$ AR polymorphism, since phenotypic effects are observed almost exlusively in Arg/Arg homozygotes. Finally, with regards to functional studies, it is always better to conduct these studies in physiologically relevant cell systems (i.e., in the Arg389Gly $\beta_{1}$ AR case, cardiac myocytes), with physiology as close to human as possible, and which express physiologically relevant levels of the (receptor) variants. Heterologous murine or rat cell lines, transfected to express supraphysiological $\beta_{1} \mathrm{AR}$ variant levels, most likely do not represent true human cardiomyocytes with endogenous $\beta_{1}$ AR levels very well.

\section{References}

1 Lymperopoulos A, Garcia D, Walklett K. Pharmacogenetics of cardiac inotropy. Pharmacogenomics 15(14), 1807-1821 (2014).

2 Ahles A, Engelhardt S. Polymorphic variants of adrenoceptors: pharmacology, physiology, and role in disease. Pharmacol. Rev. 66(3), 598-637 (2014).

3 Dorn GW 2nd. Adrenergic signaling polymorphisms and their impact on cardiovascular disease. Physiol. Rev. 90(3), 1013-1062 (2010).

4 Liggett SB, Mialet-Perez J, Thaneemit-Chen S et al. A polymorphism within a conserved beta(1)-adrenergic
Given all of these hurdles above, it should come as no surprise that the Arg389Gly $\beta_{1} \mathrm{AR}$ polymorphism has not been able to be definitively associated with heart failure, hypertension or other heart diseases, and it may very well be unrealistic to expect it ever will. Nevertheless, and despite the caveats and difficulties in interpreting and reconciling the ever-increasing number of complex studies in this field, the following conclusions about the presence of glycine (instead of arginine) at position 389 of the human $\beta_{1}$ AR can be drawn with a significant amount of certainty: Gly389 results in impaired efficacy of the $\beta$-blocker treatment in chronic heart failure (and in other heart disease) patients, and Gly389 results in reduced $\beta_{1} \mathrm{AR}$ function and signaling in vitro, as well as in suppressed cardiac and blood pressure responses of the $\beta_{1} \mathrm{AR}$ in vivo. Thus, it is quite plausible that, soon, $\beta_{1} \mathrm{AR}$ genotyping of the cardiovascular patient for the Arg389Gly variation will allow for personalization of his/ her drug therapy, since the presence of Arg389 homozygosity will identify the patient as a good responder to $\beta$-blockers (or $\beta$-agonists/cardiostimulants), whereas Arg/Gly heterozygosity or Gly389 homozygosity will signal to the clinician that a dose escalation of the prescribed $\beta$-blocker (or $\beta$-agonist drug) is necessary. The ball is now in the court of researchers performing very large GWAS's to firmly establish these inferences, so that they can be incorporated into the clinical practice sooner rather than later. The hope is that another success story for pharmacogenomics will soon be delivered, this time for the benefit of cardiology.

\section{Financial \& competing interests disclosure}

The authors have no relevant affiliations or financial involvement with any organization or entity with a financial interest in or financial conflict with the subject matter or materials discussed in the manuscript. This includes employment, consultancies, honoraria, stock ownership or options, expert testimony, grants or patents received or pending, or royalties.

No writing assistance was utilized in the production of this manuscript. receptor motif alters cardiac function and beta-blocker response in human heart failure. Proc. Natl Acad. Sci. USA 103(30), 11288-11293 (2006).

5 Mialet Perez J, Rathz DA, Petrashevskaya NN et al. Beta1adrenergic receptor polymorphisms confer differential function and predisposition to heart failure. Nat. Med. 9(10), 1300-1305 (2003).

6 Rochais F, Vilardaga JP, Nikolaev VO, Bünemann M, Lohse MJ, Engelhardt S. Real-time optical recording of beta1-adrenergic receptor activation reveals supersensitivity of the Arg389 variant to carvedilol. J. Clin. Invest. 117(1), 229-235 (2007). 
7 Sandilands AJ, O'Shaughnessy KM, Brown MJ. Greater inotropic and cyclic AMP responses evoked by noradrenaline through Arg389 beta 1-adrenoceptors versus Gly389 beta 1-adrenoceptors in isolated human atrial myocardium. Br. J. Pharmacol. 138(2), 386-392 (2003).

8 Ahles A, Rodewald F, Rochais F, Bünemann M, Engelhardt $S$. Interhelical interaction and receptor phosphorylation regulate the activation kinetics of different human $\beta 1$ adrenoceptor variants. J. Biol. Chem. 290 (3), 1760-1769 (2015).

9 Capote LA, Mendez Perez R, Lymperopoulos A. GPCR signaling and cardiac function. Eur. J. Pharmacol. doi:10.1016/j.ejphar.2015.05.019 (2015) (Epub ahead of print).

10 Wang H, Liu J, Liu K et al. $\beta 1$-adrenoceptor gene Arg389Gly polymorphism and essential hypertension risk in general population: a meta-analysis. Mol. Biol. Rep. 40(6), 4055-4063 (2013).

11 Horikoshi M, Yaghootkar H, Mook-Kanamori DO et al. New loci associated with birth weight identify genetic links between intrauterine growth and adult height and metabolism. Nat. Genet. 45(1), 76-82 (2013).

12 Yilmaz A, Kaya MG, Merdanoglu U, Ergun MA, Cengel A, Menevse S. Association of beta- 1 and beta- 2 adrenergic receptor gene polymorphisms with myocardial infarction. J. Clin. Lab. Anal. 23(4), 237-243 (2009).

13 Fragoso JM, Rodríguez-Pérez JM, González J et al. Beta1adrenergic receptor gene polymorphisms in Mexican patients with idiopathic dilated cardiomyopathy. Exp. Mol. Pathol. 80(3), 279-282 (2006).
14 Iwai C, Akita H, Shiga N et al. Suppressive effect of the Gly389 allele of the beta1-adrenergic receptor gene on the occurrence of ventricular tachycardia in dilated cardiomyopathy. Circ. J. 66(8), 723-728 (2002).

15 Kumar A, Pandit AK, Vivekanandhan S, Srivastava MV, Tripathi M, Prasad K. Association between beta-1 adrenergic receptor gene polymorphism and ischemic stroke in North Indian population: a case control study. J. Neurol. Sci. 348(1-2), 201-205 (2015).

16 Huntgeburth M, La Rosée K, ten Freyhaus $\mathrm{H}$ et al. The Arg389Gly $\beta 1$-adrenoceptor gene polymorphism influences the acute effects of $\beta$-adrenoceptor blockade on contractility in the human heart. Clin. Res. Cardiol. 100(8), 641-647 (2011).

17 Kindermann M, Seeland U, Ruhnke P, Böhm M, Maack C. Functional effects of $\beta_{1}$-adrenoceptor polymorphisms on the hemodynamic response to dobutamine with and without $\beta$-blocker administration. Clin. Res. Cardiol. 100 (2), 129-137 (2011).

18 Bruck H, Leineweber K, Temme T et al. The Arg389Gly beta1-adrenoceptor polymorphism and catecholamine effects on plasma-renin activity. J. Am. Coll. Cardiol. 46(11), 2111-2115 (2005).

19 Liu WN, Fu KL, Gao HY et al. $\beta 1$ adrenergic receptor polymorphisms and heart failure: a meta-analysis on susceptibility, response to $\beta$-blocker therapy and prognosis. PLoS ONE 7(7), e37659 (2012).

20 Taylor MR, Sun AY, Davis G, Fiuzat M, Liggett SB, Bristow MR. Race, common genetic variation, and therapeutic response disparities in heart failure. JACC. Heart Fail. 2(6), 561-572 (2014). 